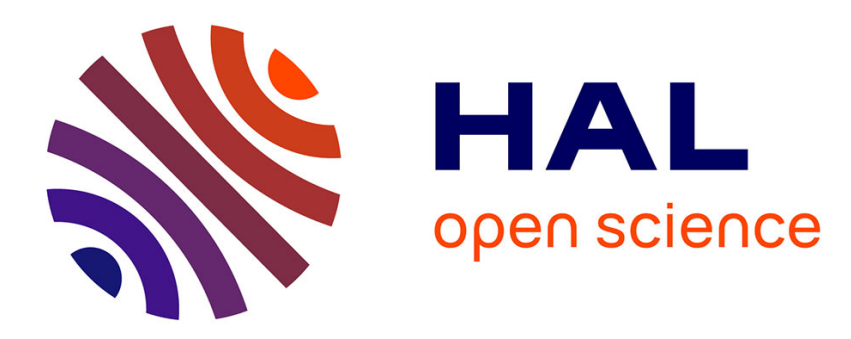

\title{
Justice familiale et migration : de la diversité culturelle à la double appartenance
}

Anne Wyvekens

\section{To cite this version:}

Anne Wyvekens. Justice familiale et migration: de la diversité culturelle à la double appartenance. Recherches familiales, 2016, Famille et migration, 96 (13), pp.65-77. halshs-01328104

\section{HAL Id: halshs-01328104 https://shs.hal.science/halshs-01328104}

Submitted on 21 Dec 2017

HAL is a multi-disciplinary open access archive for the deposit and dissemination of scientific research documents, whether they are published or not. The documents may come from teaching and research institutions in France or abroad, or from public or private research centers.
L'archive ouverte pluridisciplinaire HAL, est destinée au dépôt et à la diffusion de documents scientifiques de niveau recherche, publiés ou non, émanant des établissements d'enseignement et de recherche français ou étrangers, des laboratoires publics ou privés. 


\title{
JUSTICE FAMILIALE ET MIGRATION : DE LA DIVERSITÉ CULTURELLE À LA DOUBLE APPARTENANCE
}

\author{
Anne Wyvekens
}

À partir d'une enquête menée dans deux tribunaux de grande instance, l'article se propose de montrer comment la justice familiale, lorsqu'elle est confrontée à des justiciables immigrés ou d'origine immigrée, met en scène, plus qu'une différence entre « eux» et «nous», une double appartenance, produit complexe de la migration. Annulations de mariage et changements de prénom font voir des justiciables qui sont à la fois d'ici et d'ailleurs. Quant aux magistrats, ils abordent ces situations en naviguant entre un traitement pragmatique - relativement indifférent aux différences - des situations individuelles, et des représentations parfois suspicieuses de l'immigration en tant que phénomène collectif.

S'interroger, comme on le fait encore peu en France, sur les rapports entre la justice et la "diversité culturelle $»^{[1]}$, conduit assez naturellement à s'intéresser à la justice familiale. Crimes d'honneur, mariages forcés, polygamie et répudiations alimentent la représentation de structures familiales patriarcales et autoritaires qui posent nécessairement question au droit familial français et aux juges chargés de l'appliquer. Plus récemment, la pratique de la religion musulmane, parfois plus revendiquée, apparaît susceptible elle aussi de s'inviter sur la scène de la justice familiale. «À tort ou à raison ${ }^{[2]}$, les relations familiales se profilent comme l'un des terrains privilégiés [...] des réflexions et des discours publics traitant de la diversité culturelle et des enjeux auxquels elle confronte les systèmes juridiques occidentaux. "[3] $^{[3}$

La recherche dont il va être question dans cet article ${ }^{[4]}$ a été engagée à partir de cette intuition. Il ne s'agissait pas, précisons-le, d'examiner les modalités de mise en œuvre du droit international privé. En effet, ce prisme n'est pas le plus révélateur de la question de la diversité, dans la mesure

[1] Voir par exemple : Gérard BOUCHARD, Charles TAYLOR, Fonder l'avenir. Le temps de la conciliation. Commission de consultation sur les pratiques d'accommodements reliés aux différences culturelles, Archives Nationales du Québec, 2008, www. accommodements.qc.ca ; Doriane L. COLEMAN, «Individualizing Justice through Multiculturalism : The Liberals' Dilemma », Columbia Law Review, vol. 96, n 5, June 1996, pp. 1093-1167 ; " The Cultural Defense in the Criminal Law », Harvard Law Review, vol. 99, n 6, april 1986, pp. 1293-1311; Alison D. RENTELN, The Cultural Defense, Oxford University Press, 2004.

[2] C'est nous qui soulignons.

[3] Barbara TRUFFIN, François LAPERCHE, « "Ils emportent leur secret". Regards ethnographiques sur le traitement judiciaire des conflits conjugaux en contexte multiculturel », in J. RINGELHEIM (dir.), Le droit et la diversité culturelle, Bruxelles, Bruylant, pp. 657-698, 2011, p. 657.

[4] Justice familiale et diversité culturelle, recherche subventionnée par la Mission de recherche Droit et Justice, avril 2015. 
où une part non négligeable des couples concernés est de nationalité française et se voit donc appliquer le droit français. Ce que l'on cherche à appréhender, c'est la façon dont des valeurs et des modes de vie qui sont par hypothèse différents de ceux de la culture majoritaire sont (ou non) pris en compte par le juge. "Comment appréhender correctement les modalités d'une séparation sans chercher à connaître son cadre : la belle-mère qui vient d'arriver et ne comprend pas que la femme de son fils ne soit pas à son entier service, ledit fils préférant encore la faillite de son couple à la honte de mettre sa mère à la porte... $»^{[5]}$

Le travail de terrain s'est déroulé dans le cadre de deux tribunaux de grande instance, l'un en Île-de-France (IdF) (population très diversifiée tant sur le plan des origines que sur celui des catégories socioprofessionnelles), l'autre dans une ville moyenne du Sud-Est (SE) (importante population d'origine maghrébine, notamment marocaine). La majeure partie du recueil d'informations, de type qualitatif, a consisté dans des observations d'audiences (13 audiences à juge unique et 3 audiences collégiales à $\mathrm{SE}, 12$ audiences à juge unique et 2 audiences collégiales à IdF) et des entretiens avec des magistrats ( 9 entretiens avec des juges aux affaires familiales, et 2 entretiens au parquet civil à SE, 6 entretiens JAF et 1 entretien au parquet civil à IdF) (précision : tous les magistrats interrogés étaient des magistrates).

Un dépouillement de jugements rendus en matière de nullité de mariage et de changement de pré-

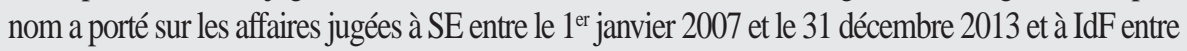
le $1^{\text {er janvier }} 2009$ et le 31 décembre 2013. En l'absence d'indications dans les bases de données sur la nationalité ou l'origine des parties, la sélection des affaires impliquant des justiciables d'origine immigrée a été réalisée à partir des noms et prénoms des parties : à SE, 34 annulations de mariage et 65 changements de prénom; à IdF, 116 annulations de mariage et 118 changements de prénom.

Parce que les parties y sont présentes en personne, et que la procédure est orale, nous avions choisi au départ d'assister aux audiences à juge unique ayant pour objet la conciliation avant divorce et les mesures après-divorce. Alors que ce terrain paraissait idéal pour observer la façon dont la justice familiale met en scène et dont les magistrats abordent la « diversité », la récolte s'est avérée limitée : lorsque les couples d'origine immigrée divorcent, leurs demandes ne diffèrent guère - les magistrats le confirment - de celles des autres couples. Lorsque cette « diversité » apparaît, elle n'est pas au centre du débat. Fausse piste ? Non. Au-delà de cette absence, analysée comme une conjugaison de silences ${ }^{[6]}$, la poursuite de l'enquête a conduit à un déplacement du regard, à une autre construction de l'objet. Si les demandes formulées au cours des procédures de divorce ou d'après-divorce ne présentent pas souvent de particularités, on a constaté que d'autres procédures relevant de la compétence des juges aux affaires familiales avaient pour protagonistes, en majorité, des justiciables d'origine immigrée. Il s'agit d'une part des requêtes en annulation de mariage, d'autre part des demandes de changement de prénom. On voudrait montrer, à partir de là, d'une part comment la justice familiale met en scène, plus qu'une différence plus ou moins irréductible, une double appartenance, produit complexe de la migration et, d'autre part, comment les juges aux affaires familiales abordent cette réalité empirique différente de celle des représentations stéréotypiques évoquées au départ.

[5] France BLANMAILLAND, « Droit familial et diversité culturelle : un point de vue de praticienne, in J. RINGELHEIM (dir.), Le droit et la diversité culturelle, op. cit., pp. 713-722, p. 720.

[6] Anne WYVEKENS, Justice familiale et diversité culturelle, rapport cité. 


\section{_."D'ici etd'ailleurs: de la diversité à la double appartenance}

Ce que l'on voit en justice familiale est tout aussi instructif que ce que l'on n'y voit pas. Des demandes se révélant spécifiques aux justiciables d'origine immigrée existent bel et bien, mais ailleurs que là où on les attendait. Leur objet les rattache moins à la culture des justiciables concernés qu'à leur origine étrangère et au phénomène de la migration en tant que tel.

\section{- Venir d'ailleurs : mariage et migration}

Le contentieux des annulations de mariage, pour réduit qu'il soit en quantité, s'avère particulièrement intéressant pour aborder la « diversité ». Ses protagonistes sont en effet, dans l'écrasante majorité des cas, des personnes d'origine immigrée. À quoi correspond cette surreprésentation ? Le discours des magistrats fait apparaître deux éléments principaux, de nature - et de portée différente. Ils qualifient le premier de « culturel » : le caractère stigmatisant du divorce conduirait certains couples d'origine maghrébine à demander l'annulation de leur mariage plutôt que d'y mettre fin par un divorce. L'autre enjeu, que nous avons qualifié d'administratif, concerne l'argument le plus fréquemment invoqué (que ce soit par le parquet ou par l'un des conjoints) pour obtenir l'annulation : le mariage aurait eu pour unique objectif l'obtention d'un titre de séjour. Les deux enjeux s'entrecroisent souvent.

Ici comme en matière de divorce, les dossiers le montrent, l'élément « culturel » demeure implicite : les magistrats le connaissent, en parlent d'abondance, mais les jugements sont - sans grande surprise - muets sur ce point. Seul l'argument « administratif » est susceptible d'apparaitre au grand jour, puisqu'il constitue le fondement juridique de la demande ${ }^{[7]}$. Quand il s'agit de «mariage pour les papiers », la requête est introduite en général sur le fondement de l'absence de consentement (art. 146, C. civ.), un des époux accusant l'autre (ou le parquet accusant un époux ou les deux) d'avoir été, au moment du mariage "dépourvu d'intention matrimoniale », en l'occurrence de s'être marié en ayant pour objectif non pas une vie conjugale mais uniquement l'obtention d'un titre de séjour.

La plupart des magistrates interrogées ont à ce sujet un discours dépourvu d'équivoque. Le lien entre mariage et migration représente une évidence. L'une d'entre elles évoque « une porte d'entrée dans le flux migratoire », une autre "un sport local ». L'examen de ces dossiers, qui déroulent l'histoire de couples dont l'un des conjoints est socialisé en France, et l'autre venu " $d u$ bled », en dit long sur ceux-là, et éclaire également un certain nombre d'audiences de conciliation avant divorce. Le détour par le contentieux - marginal - des annulations de mariage rend visible, explicite, ce qui n'apparait que fugacement, voire pas du tout, dans de nombreux cas de divorce.

La justice familiale offre ainsi une autre image, plus complexe, des couples d'origine immigrée. Elle apparaît d'autre part comme une scène où la double appartenance de ces justiciables conduit à des « jeux avec les codes ».

[7] On n'évoque pas ici les autres motifs d'annulation - moins fréquents et moins souvent évoqués par les magistrats - apparus à l'occasion de l'examen des jugements concernant des personnes d'origine immigrée. Deux autres catégories de motifs apparaissent essentiellement, l'un de fond - la bigamie -, l'autre de forme - l'incompétence de l'officier d'état civil ayant célébré le mariage (mariages célébrés au consulat le plus souvent). 


\section{- Mariages blancs, mariages gris, mariages arrangés}

Se marier « pour les papiers » n'équivaut pas nécessairement à faire un mariage blanc. Les véritables mariages blancs - ceux où aucun des deux conjoints n'a de véritable intention matrimoniale, l'opération s'accompagnant ou non du versement d'une somme d'argent - font même figure d'exception dans l'échantillon de jugements. Cette « absence » est moins le reflet d'une quelconque réalité que d'éléments liés à l'organisation et au fonctionnement de l'institution judiciaire. D'une part, il s'agit le plus souvent d'actions intentées par le parquet, et non par les parties : leur nombre dépend donc de la politique du parquet en la matière. Le mariage blanc est, d'autre part, difficile à prouver, ce qui rend les parquets, même plus enclins à poursuivre, particulièrement circonspects.

Il ne s'agit pas non plus nécessairement de mariages mixtes au sens où le conjoint français serait dépourvu de parcours ou d'ascendance migratoire. Au contraire, les magistrates évoquent plus souvent des unions entre personnes de nationalité différente mais d'origine étrangère identique, dont l'une vit de façon régulière en France et l'autre émigre à l'occasion du mariage. Le dépouillement des jugements confirme cette prévalence. Ces couples « mixtes » renvoient à la complexité des définitions de la mixité, une fois que le point de vue adopté s'écarte aussi bien du sens commun (le « couple domino $»^{[8]}$ ) que d'une approche strictement juridique, fondée sur la nationalité. "Seule l'articulation de la dimension juridique [nationalité] avec la dimension ethnocul-

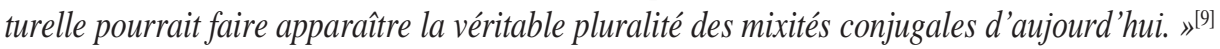
S'agit-il de mariages arrangés? Parfois, ou alors « semi-arrangés, qui ont au moins la validation de la famille, et sont célébrés dans le pays d'origine » ${ }^{[10]}$. Mais il s'agit également de choix personnels. Les magistrates, interrogées au fil des audiences, ont une vision souvent très précise de ces histoires de couples. Elles racontent, à leur manière, ce que décrivent et analysent anthropologues et sociologues $^{[11]}$. Parfois, un époux a été berné par l'autre, parfois simplement, cela aurait pu marcher mais la différence culturelle en décide autrement. Il n'est pas toujours facile de faire la part entre les véritables « escroqueries sentimentales à but migratoire " ${ }^{[12]}$ et ces mariages arrangés à l'ancienne, qui auraient pu durer, mais où les relations ont fini par se tendre et la vie conjugale devenir intolérable.

Le regard se déplace : le « conflit de cultures » existe bien, mais ailleurs que là où on s'attendait à le trouver : non pas entre les valeurs des justiciables d'origine étrangère et celles du pays d'accueil mais à l'intérieur même de certains couples ou familles de même origine. Si ces couples se séparent, ce n'est pas uniquement parce que l'un reproche - de plus ou moins bonne foi - à l'autre de ne l'avoir épousé « que pour les papiers », c'est qu'ils sont trop différents.

[8] Expression de Catherine PHILIPPE (Le couple domino et bigarré, DEA, EHESS, Paris, 1983), rappelée par Gérard NEYRAND et Marine M'SILI, «Les couples mixtes dans la France contemporaine. Mariage, acquisition de la nationalité française et divorce », Population, vol. 52, n 3, 1997, pp. 571-605.

[9] Beate COLLET, « Pluralité contemporaine de la mixité conjugale et position asymétrique entre les sexes », Diasporas : Transports amoureux, $\mathrm{n}^{\circ} 15$, p. 53-67, 2010, p. 61.

[10] Entretien, JAF, IdF.

[11] Cf. par exemple Beate COLLET, Emmanuelle SANTELLI, «La mixité au-delà des différences culturelles. Les choix conjugaux des descendants d'immigrés maghrébins, in Beate COLLET, Claudine PHILIPPE (dir.), Mixité(s). Variations autour d'une notion transversale, L'Harmattan, 2008. Ou encore Leyla ARSLAN, Enfants d'Islam et de Marianne. Des banlieues à l'université, PUF, 2010, p. 258.

[12] L'expression, lue dans une requête, a en fait été utilisée en 2009 par Éric Besson, alors ministre de l'Immigration, de l'Intégration, de l'Identité nationale et du Développement solidaire. Elle reprend le titre du livre de M.-A. DELAUNAY, L'immigration par escroquerie sentimentale, Tatamis, 2006 (source : Anne UNTERREINER, Enfants de couples mixtes. Liens sociaux et identités, Presses universitaires de Rennes, 2015. 
Une psychologue belge, Jamila Moussaoui, a forgé, concernant ces couples, marocains en l'occurrence, le concept d' "endomixité » ${ }^{[13]}$. "Donner un nom spécifique semblait urgent afin de répondre à l'inconfort verbal et écrit généré mais aussi afin de rendre compte de la dynamique interne particulière à ces couples, une dynamique qui conjugue à la fois un écart et un rapprochement. » Son point de vue de clinicienne est éclairant, il explicite ce que l'on a pu pressentir à l'observation des audiences : "le doute [qui] envahit les esprits et vient empoisonner la relation conjugale ", "l'épreuve de la distance culturelle », la distance des conjoints par rapport à leur culture d'origine n'étant pas la même pour l'un et pour l'autre ; deux épreuves qui viennent s'ajouter à celle, initiale, de l'exil. L'anthropologue Pascale Jamoulle aborde également cette question, à laquelle elle consacre de belles pages ${ }^{[14]}$. Elle enchaîne, comme Jamila Moussaoui, sur les conséquences de ces unions, le sentiment d'humiliation, et parfois le désir de vengeance que les magistrats décrivent ou qu'on a pu deviner en arrière-plan de certaines procédures de divorce ou d'annulation de mariage ${ }^{[15],[16]}$.

On s'est arrêté sur ces couples, car ils cristallisent ce que donne à voir, plus généralement, la justice familiale : la rencontre avec des personnes dont la caractéristique n'est pas tant une sorte d'altérité irréductible qu'une combinaison d'éléments divers, résultat de la migration, constitutive de ce que l'on a qualifié de "double appartenance ", et les tensions qu'elle comporte y compris à l'intérieur des familles elles-mêmes. La dimension « culturelle » n'est pas absente, mais elle n'a pas l'homogénéité que lui confèrent les stéréotypes. Et elle s'accompagne d'autres caractéristiques, de nature juridique, administrative, géographique, ainsi qu'en témoigne par exemple la seule demande spécifique aux justiciables d'origine étrangère que l'on observe fréquemment devant les JAF : le prononcé d'une interdiction de quitter le territoire.

Un avocat belge qui pratique la médiation familiale interculturelle et internationale résume le fruit de son expérience: "Pour tous, il s'agit de trouver sa place dans un monde en profonde mutation, de se construire une existence tout en acceptant l'inconfort d'un irréductible entre-deux. $»^{[17]}$

\section{- «Jouer avec les codes »}

La mise en évidence du phénomène de double appartenance laisse entrevoir, d'autre part, les stratégies que les justiciables peuvent mettre en œuvre, jouant tantôt d'un « code », tantôt d'un autre, naviguant entre impératifs " culturels », droit et justice d'un univers et de l'autre. Obtenir - de la justice française - l'annulation du mariage peut être une façon de satisfaire aux exigences de «l'autre code », culturel, qui stigmatise le divorce. Les époux ont bel et bien vécu ensemble, ils ont même parfois eu un enfant, mais l'annulation effacerait cet épisode. Les magistrats le savent:

"Quand ça rate, ils viennent dire qu'ils veulent annuler leur mariage en disant que l'autre n'était intéressé que par les papiers. De fait, il a eu les papiers, mais est-ce qu'au départ il n'était pas d'accord pour essayer? Avec en plus ce critère particulier dans

[13] Jamila MOUSSAOUI, « Mariages endomixtes marocains », L'Agenda interculturel, n 245, 2006, pp. 10-13.

[14] Pascale JAMOULLE, Par-delà les silences. Non-dits et ruptures dans les parcours d'immigration, La Découverte, 2013, p. 108.

[15] Pascale JAMOULLE, op. cit., p. 119.

[16] Voir également Nawal BENSAÏD, «La politique migratoire belge et ses conséquences sur les couples transnationaux : un regard des acteurs sociaux bruxellois », in Migrations Société, vol. 25, $\mathrm{n}^{\circ}$ 150, 2013, dossier Mariages et migrations : l'amour et ses frontières, pp. 109-121, p. 114 et s.

[17] Damien D’URSEL, La médiation, entre tradition et modernité familiales, Presses universitaires de Louvain, 2010, p. 78. 
cette communauté, qu'il faut annuler le mariage et non pas divorcer. J'ai eu quelques cas où on comprend que c'est juste parce qu'on veut effacer le mariage pour garder une "virginité" à l'état civil, pour pouvoir se remarier. C'est important au point de saisir un avocat, avec ce que ça coûte. » (entretien, parquet civil, SE).

Certains jugements en témoignent. Ainsi, dans ce dossier où c'est le mari, français d'origine algérienne, qui demande l'annulation. Les époux sont des cousins éloignés. Le mariage a été célébré en Algérie, à la suite de quoi l'épouse a obtenu un visa et s'est installée en France. Les éléments reportés dans le jugement font apparaître que les relations se sont progressivement dégradées. Le mari demande la nullité, sur la base de l'article 146 du code civil, l'épouse ne s'y oppose pas : les deux époux, ici, choisissent de fait la voie de l'annulation (qui leur sera refusée), dans une situation relevant en réalité d'une procédure de divorce.

"L'examen [des] pièces révèle que les époux ne s'entendent plus et que l'épouse est d'accord pour une dissolution de leur mariage à condition de ne pas être contrainte de retourner en Algérie avec le statut de "divorcée", à l'âge de 23 ans. Mais l'examen de ces pièces ne permet nullement d'établir que Madame était dépourvue de toute intention matrimoniale lors de la célébration du mariage. » (extrait du jugement).

Une forme de « jeu » de ce type est observable également au cours de procédures en divorce. À l'audience, un homme demandant le divorce insiste sur le fait que son mariage était "arrangé ». Il l'était sans doute plus ou moins, avec une jeune femme venue du pays et cela... « arrangeait » bien l'un et l'autre des conjoints. Jusqu'au jour où l'époux a rencontré une autre femme et cherché, devant la justice française, à se débarrasser de la première. Il jouait à présent du côté de l'autre culture, celle du juge, celle du pays d'accueil, celle pour laquelle le mariage arrangé relève de l'archaïsme. Son épouse, elle, disait vouloir " sauver son mariage », en réclamant une médiation... histoire probablement qu'il subsiste jusqu'à l'obtention du titre de séjour.

Sur un plan strictement juridique, enfin, on a vu comment « jouer avec les codes » peut consister, en général pour les maris, à répondre à une requête en divorce introduite en France par l'introduction d'une requête dans le pays d'origine, pour se voir appliquer une loi supposée plus favorable.

\section{- Être d'ici et d'ailleurs : le choix du prénom}

Un autre type de requête relevant de la compétence de la chambre de la famille a retenu notre attention. Ce sont les demandes de changement de prénom. De façon assez surprenante, plusieurs magistrates d'une des juridictions enquêtées, interrogées sur les particularités qu'elles observaient chez les justiciables d'origine immigrée, présentaient cette question comme leur paraissant particulièrement problématique.

Tout comme les annulations de mariage, les requêtes en changement de prénom sont peu nombreuses, mais elles impliquent un nombre relativement important - et qui serait croissant - de justiciables d'origine étrangère. Ces requêtes ont longtemps porté (et portent encore) pour ceuxci sur le remplacement de leur prénom « d'origine » par un prénom français. L'intérêt légitime exigé par la loi (art. 60, C. civ.) consiste alors à plaider le souci d'intégration et/ou à évoquer des difficultés ou des discriminations dans l'accès à l'emploi ou au logement. Mais les juges aux affaires familiales se souviennent surtout des demandes « en sens inverse » : celles où une 
personne d'origine étrangère portant un prénom français demande à ce que lui soit officiellement attribué un prénom renvoyant à sa culture d'origine. Il peut s'agir, mais pas toujours, de ce que Baptiste Coulmont appelle des "défrancisations » ${ }^{[18]}$ : des personnes qui, au moment de leur naturalisation, avaient choisi de troquer leur prénom d'origine contre un prénom français (parfois dans l'espoir de faciliter l'obtention de la nationalité française $\left.{ }^{[19]}\right)$, puis regrettent ce changement et souhaitent rétablir l'état d'origine. Il s'agit parfois aussi de jeunes d'origine étrangère auxquels leurs parents venus s'installer en France ont donné, à la naissance, un prénom français, et qui, une fois majeurs, s'adressent au tribunal pour se voir attribuer un prénom correspondant à leur origine. La demande peut également être introduite par des parents à propos de leur enfant mineur. Toutes ces demandes auraient, selon les JAF, «explosé ». L'examen d'un échantillon de dossiers montre qu'elles demeurent moins nombreuses que les demandes de francisation. Mais, comme l'a également constaté Baptiste Coulmont ${ }^{[20]}$, «ce sont des cas problématiques » qui dès lors « "occupent" et l'esprit et le temps des magistrats».

De la même manière qu'à l'occasion des ruptures conjugales, on voit ici des enjeux encodés par les magistrats comme «culturels » s'entrecroiser avec des enjeux d'ordre administratif. Il est question, d'une part, d'identité (parfois de religion). Il s'agit du besoin éprouvé par le demandeur de ne pas se sentir étranger, voire rejeté au sein de sa famille ou de sa communauté. «Les jeunes, et c'est la majorité des dossiers, c'est plutôt parce qu'ils le vivent mal, pour des problèmes d'intégration à leur communauté, à cause de moqueries de l'entourage, parce qu'ils sont les seuls de la fratrie... » ${ }^{[2]} \mathrm{Le}$ " problème identitaire » invoqué à l'appui de ces demandes prend parfois des proportions quasi-pathologiques. Plusieurs juges le relèvent, et on en trouve confirmation dans certains dossiers, qui mentionnent la présentation, à l'appui de la demande, de certificats médicaux. Parfois, le demandeur indique s'être converti à l'islam, ou avance la nécessité de porter un prénom « musulman » pour pouvoir effectuer le pèlerinage à La Mecque.

Une seconde catégorie de demandes de changement de prénom ou de " défrancisation » est liée de façon administrative à la double appartenance nationale des personnes issues de l'immigration. Ces demandes sont motivées par le fait que les autorités du pays d'origine - le Maroc en particulier - conditionnent l'octroi de certains droits, la réalisation de certaines formalités juridiques - inscription sur les listes d'état civil, acquisition de la nationalité, obtention d'un visa, liquidation d'une succession... - au fait d'avoir un prénom « marocain »"22]. On rapprochera cette préoccupation que manifeste l'État marocain de garder une emprise sur ses nationaux ${ }^{[23]} \mathrm{de}$ certaines dispositions, relatives au mariage, du nouveau droit de la famille marocain de 2004 ${ }^{[24]}$.

[18] Baptiste COULMONT, Changer de prénom. Une sociologie des usages de l'état civil, rapport pour la Mission de recherche Droit et Justice, 2013.

[19] Cf. Abdellali HAJJAT, Les frontières de l'« identité nationale ». L'injonction à l'assimilation en France métropolitaine et coloniale, La Découverte, 2012, en particulier les pages consacrées à la mesure de l'assimilation.

[20) Baptiste COULMONT, op. cit., p. 98.

[21] Entretien, parquet civil, SE.

[22] Art. 6 bis (ajouté en 1996) du texte régissant l'état civil : « Le prénom choisi par celui qui effectue la déclaration de naissance en vue de l'inscription sur le registre de l'état civil doit présenter un caractère traditionnel marocain et ne doit être ni un prénom étranger, ni un nom de famille, ni composé de plus de deux prénoms, ni un nom de ville, village ou tribu, comme il ne doit pas porter atteinte à la morale ou à l'ordre public. » Le mot « traditionnel » a été supprimé par une loi de 2002.

[23] Baptiste COULMONT, op. cit., p. 103.

[24] Marie-Claire FOBLETS, «Le nouveau droit de la famille marocain (2004) : un codificateur soucieux de ses nationaux émigrés à l'étranger ", in Christoph EBERHARD, Geneviève VERNICOS (éds), La quête anthropologique du droit. Autour de la démarche d'Étienne Le Roy, Karthala, 2006, pp. 403-422. 
Se pencher sur les changements de prénom apporte un éclairage supplémentaire à ce que l'on a découvert en examinant les divorces et les annulations de mariage. Que ce soit sous l'angle « culturel » ou sous l'angle « administratif », ces demandes montrent à leur tour que la justice familiale connait des "réalités bien plus complexes que celles tirées d'une opposition entre "nos valeurs" et celles "des autres" "[25] : la migration, la double appartenance se traduisent ici en termes d'identité. Identité choisie, parce qu'on est à la fois d'ici et d'ailleurs, identité imposée pour pouvoir demeurer à la fois d'ici et d'ailleurs. Identité complexe.

\section{_.Juger les familles venues d'ailleurs}

Ce que la justice familiale donne à voir de la « diversité » s'accompagne d'une seconde série d'enseignements, qui concernent cette justice elle-même. Le détour par ces deux contentieux spécifiques aux personnes immigrées ou d'origine immigrée éclaire la façon dont l'institution judiciaire en tant qu'institution, et les magistrats en tant qu'individus, abordent non pas tant une « autre culture » que les immigrés et l'immigration. Le mariage est une des dernières façons possibles d'accéder au territoire français. Comment la justice familiale intègre-t-elle cet élément à sa pratique ? Quant aux changements de prénom, quel est l'impact de la recrudescence de demandes de remplacement d'un prénom français par un prénom étranger?

Les deux juridictions enquêtées présentent chacune des particularités dans le traitement de ces contentieux. En matière d'annulation de mariage, le parquet de SE se contente de conclure sur les demandes que formulent les conjoints eux-mêmes, alors que celui d'IdF se montre plus proactif. Les changements de prénom s'obtiennent quasi systématiquement à IdF alors qu'ils sont longuement débattus et parcimonieusement accordés à SE. On n'abordera pas les éléments de nature institutionnelle et les facteurs locaux susceptibles d'influer sur ces pratiques. La taille de la juridiction et son engorgement, les moyens dont dispose le parquet civil, l'importance et l'origine de la population immigrée du ressort, la politique - éminemment variable - des municipalités en matière d'état civil jouent un rôle non négligeable. On s'attachera ici au discours des magistrats sur leur pratique et à ce qu'il dit de leurs représentations des justiciables immigrés, de l'immigration, et du rôle qu'ils estiment avoir à jouer.

— "Ce n'est pas de ça que nous sommes juges" "

Introduisant un dossier consacré aux migrations de mariage, Maïté Maskens évoque la suspicion, "un thème qui apparait en filigrane dans toutes les contributions [...], tant elle semble généralisée, structurant pour ainsi dire le champ de la migration de mariage ${ }^{[26]}$. Qu'en est-il dans ce lieu particulier de régulation qu'est la justice familiale ? Le dossier n'aborde la justice qu'au détour d'une phrase, négativement : «Les tribunaux ne nous suivent pas », déplorent les agents des consulats ${ }^{[27]}$.

[25] Barbara TRUFFIN, François LAPERCHE, op. cit., p. 696.

[26] Maïté MASKENS, «L'amour et ses frontières : régulations étatiques et migrations de mariage (Belgique, France, Suisse et Italie) », in Migrations Société : Mariages et migrations : l'amour et ses frontières, vol. 25, n 150, pp. 43-60, 2013, p. 54.

[27] Federica INFANTINO, « Gouverner les frontières ou appliquer des droits ? Le contrôle des mariages aux consulats de Belgique, d'Italie et de France à Casablanca, in Migrations Société, op. cit., pp. 79-94, p. 82. 
Les JAF, on l'a dit, se trouvent aux premières loges : elles voient comment les couples se défont, mais également comment ils se sont faits. Elles connaissent le phénomène, elles en donnent la mesure :

"Au moins trois dossiers sur quatre du contentieux de la chambre de la famille en matière de filiation et de droit du mariage sont des dossiers qui concernent des personnes qui ont utilisé la filiation ou le mariage pour rechercher l'établissement de droits, ou d'accès au pays. [...] En fait, les JAF régularisent plein de situations d'immigration, plus ou moins régulière, où un petit texte de loi, ou le mariage ou la filiation, ou la délégation d'autorité parentale ou la kafala, est utilisé pour permettre une arrivée, une installation, ça c'est sûr. » (entretien, JAF, SE).

Les magistrats du parquet, quant à eux, sont détenteurs du pouvoir non seulement de requérir l'annulation d'un mariage mais également, en amont, de prononcer sursis et opposition à mariage. La lutte contre les «mariages simulés », ou «mariages de complaisance », fait l'objet d'une circulaire du ministère de la Justice ${ }^{[28]}$ adressée aux chefs de juridiction, dont l'esprit consiste clairement à renforcer, tant préventivement qu'a posteriori, la lutte contre l'utilisation du mariage à des fins migratoires. Les magistrats y sont invités à informer les officiers de l'état civil de leurs prérogatives en la matière et à les encourager à en faire usage. L'accent est mis d'autre part sur les moyens d'action du parquet, avant et après le mariage. La " grille d'audition » annexée à la circulaire, destinée à « faciliter le travail préventif de l'officier de l'état civil », de même que le type d'interrogatoire à mener en cas d'enquête par la police sont emblématiques de la suspicion évoquée.

L'enquête offre un tableau assez éloigné de cette «traque » du mariage suspect. Pour autant qu'on puisse en juger à partir de deux juridictions, la lutte contre l'immigration par le mariage n'est pas au centre des priorités de la justice familiale.

Du côté des parquets, même si l'un se montre plus proactif que l'autre, les magistrates interrogées se déclarent «peu interventionnistes » en la matière. Le mariage est « une affaire entre parties » et le parquet n'a, disent-elles, vocation à intervenir qu'en cas de fraude manifeste, de trouble à l'ordre public. Elles concentrent leurs interventions sur la dimension potentiellement pénale de certaines situations, les réseaux, les abus de faiblesse. Si la suspicion existe au niveau des outils qui leur sont fournis, elles en font un usage sensiblement plus aléatoire que ce qui ressort, par exemple, des enquêtes réalisées dans les consulats ou à l'Office des étrangers en Belgique.

Maïé Maskens, observant l'audition des candidats au mariage, évoque «les attentes normatives d'amour romantique utilisées par les officiers de l'état civil lorsqu'ils évaluent un projet de mariage entre un immigrant non européen et un ressortissant belge » ${ }^{[29]}$. Pour une parquetière française, au contraire, apprécier l'existence du consentement d'époux « venus d'ailleurs » implique de «ne pas plaquer nos conceptions ». On voit comment elle a acquis, et intégré, une connaissance précise, quasi-anthropologique des situations, loin de l'ethnocentrisme suspicieux observé dans d'autres sphères.

"Le mariage, c'est une façon d'obtenir des papiers pour la personne. Elle se marie pour ça et la personne qui va "fournir les papiers" le sait aussi. Il n'est pas écrit dans le code civil que c'est l'amour qui doit... Le consentement, c'est le consentement. L'amour, c'est

[28] Circulaire CIV/09/010 du 22 juin 2010 relative à la lutte contre les mariages simulés (dite « circulaire Alliot-Marie »).

[29] Maité MASKENS, "Screening for Integration and Romance in Brussels' Immigration Office”, Berghan Publisher (à paraître). 
autre chose. Il ne faut pas confondre les deux. Ce n'est pas que le parquet ne veut pas intervenir, c'est qu'on est sur des notions qui sont des notions juridiques, précises, il ne faut pas y mettre des clichés, des idées qu'on a sur... ce que doit être un mariage d'amour dans nos sociétés occidentales et qui n'est pas forcément ce qui fonde un mariage pour des personnes de culture différente, mais où il y a quand même un consentement qui... qui n'est pas fondé sur les mêmes choses mais qui est un consentement quand même. [...] Ils vont au bled pendant l'été. Si j'ai bien compris, c'est une recherche de conjoint. Ils se rencontrent, ils se mettent plus ou moins d'accord et après... Soi-disant ils communiquent par téléphone, mais ils ne vont pas se voir pendant un an et préparer le mariage. Ça nous paraît suspicieux dans notre "culture occidentale”. Pour eux, il n'y a pas forcément le sentiment amoureux, mais ce n'est pas de ça dont nous sommes juges. Il y a l'intention de se marier, pour s'intégrer, pour être une femme mariée, pour être marié avec quelqu'un du même village que la famille, etc. Même si au niveau des cultures, ça peut heurter, juridiquement, ça ne démontre rien. Il y a parfois des choses qui paraissent bizarres aux officiers d'état civil. Dans notre culture, c'est effectivement bizarre, mais ce n'est rien d'autre que bizarre et différent. Même s'il n'y a pas de sentiments, on n'est pas juges de tout ça, il y a une intention matrimoniale. Après, ça se passe plus ou moins bien... » (entretien, parquet civil, SE).

Quant aux JAF, au cours des audiences de conciliation ou d'homologation de divorce par consentement mutuel, les « dessous » des mariages ne font l'objet d'aucun commentaire. Lors de la première partie de l'enquête, une présidente de chambre civile était catégorique : "Notre travail est de faire de l'exequatur, pas de la lutte contre l'immigration. »Ou, comme l'explicite un magistrat détaché au service central de l'état civil à Nantes, il faut distinguer protection du mariage et lutte contre l'immigration : "La notion de "complaisance" ou mariage "gris" ou "blanc" n'existe pas dans le code civil [...], c'est une approche migratoire. " ${ }^{[30]}$ "Un outil de droit civil ne doit pas être un outil de lutte contre les mécanismes migratoires. Son objectif principal est de protéger l'institution du mariage [...]. Sinon, on pervertit les outils. $»^{[31]}$ La parquetière interrogée à $\mathrm{IdF}$, comparant sa pratique à celles de ses collègues, suggérait à ce propos que leur propension à poursuivre en annulation les mariages suspects était liée à leur conception personnelle du mariage. Si la suspicion existe, c'est « en sourdine », sans influence sur le traitement du litige. Ainsi, chez cette JAF qui a pour habitude, lorsque le divorce met fin à un mariage manifestement « migratoire », de congédier les époux sur un : "La prochaine fois que vous vous mariez, réfléchissez! » Et d'ajouter, à l'intention du chercheur :

"Je n'aime pas qu'on me prenne pour une idiote. Je leur fais toujours comprendre à l'audience que je ne suis pas dupe. Le mariage c'est quelque chose d'important. » (entretien, JAF, IdF).

Une autre JAF ne dit pas autre chose, en se mettant, elle, à la place des justiciables.

"C'est rigolo, les gens nous font confiance. On pourrait très bien aller voir le procureur en disant: "J'ai vu un sacré truc de fraude à la loi." En général, au moins un des conjoints exprime que le mariage a été une technique plus qu'une union recherchée, voulue et vécue. » (entretien, JAF, SE).

[30] Cité par Federica INFANTINO, op. cit., p. 81.

[31] Ibid., p. 92. 
Cette distance se traduit également en creux. Qu'il s'agisse des conséquences qu'un divorce ou une annulation peut avoir en matière de séjour, ou de questions plus générales concernant la législation des étrangers, la plupart des magistrates interrogées reconnaissent leur ignorance. Et, pour l'une d'elles, il n'est de toute évidence pas le moins du monde question d'alerter le parquet.

"Quand on est saisi d'une procédure de divorce on ne va pas alerter le parquet en disant "Là il faut une annulation". [...] Je me demande qui saisit le parquet. Ou c'est quelqu'un qui écrit. [...] Je pense qu'à la préfecture, pour faire leur titre de séjour, les gens doivent justifier d'où ils habitent, etc., donc après... Les textes ont beaucoup changé. Au début, la communauté de vie, il fallait un an, puis deux ans, voilà. Donc peut-être que la préfecture [...] parce qu'ils font des enquêtes. C'est un sujet que je ne maîtrise pas du tout, je ne pourrais pas vous dire. » (entretien, JAF, SE).

Une seule JAF soutient un point de vue « interventionniste »- sur fond d'une présentation d'elle-même comme une juge atypique. Issue de l'administration fiscale, elle attribue à sa formation initiale l'importance qu'elle accorde d'abord à « la chasse au gaspi », ensuite, plus largement, à la dénonciation de toute irrégularité, quelle qu'elle soit. Elle trouverait donc naturel de signaler au procureur, voire à la préfecture, les mariages annulés en raison de leur caractère « blanc » ou « gris ».

\section{¿ "C'est quoi le message? Vous n'êtes ni français ni marocain?"}

Si la question des changements de prénom se pose dans les deux juridictions, son traitement n'est problématique que dans l'une d'elles, à SE. À IdF, les magistrats, au parquet comme au siège, voient là « les rares dossiers où l'on fait plaisir aux gens », et ajoutent "ça prend trente secondes et ça fait un dossier de plus ». On s'arrêtera au débat qui anime la première.

Les demandes motivées par les exigences de l'État marocain sont particulièrement mal vécues. L'intérêt du demandeur est légitime, mais certains magistrats jugent inacceptable ce qu'ils considèrent comme une ingérence de l'État marocain dans la vie de citoyens français, voire dans les prérogatives de l'État français. Les demandes « identitaires » ne sont pas systématiquement reçues avec plus de bienveillance, en particulier celles qui visent à reprendre, après francisation, un prénom d'origine. L'argument consiste alors à déclarer protéger la stabilité de l'état civil, qui "n'est pas une carte de restaurant ${ }^{\text {"32] }}$.

Les propos contrastés de deux magistrates illustrent la polarisation qui anime le débat. Pour l'une, c'est l'image de la société française qui est en jeu. Outre une sensibilité forte à l'ingérence de l'État marocain, elle s'inquiète d'une mutation de la société, et on voit s'expliciter, en toile de fond de son discours, une conception de l'immigration en termes de menace, «des communautés avec lesquelles on a plus de problèmes que d'autres ».

"Moi je m'en fous si dans cinquante ans, il y a cinq prénoms sur dix qui sont d'origine musulmane, ça ne changera pas le mien, ça ne changera pas ceux de mes enfants, on n'aura pas plus ou moins de choix... mais quelle image on veut donner aussi de notre société, sachant ce que ça implique derrière ? [...] Il ne faut pas se voiler, il y a des communautés avec lesquelles on a plus de problèmes que d'autres. [...] Soit on considère que la société française, elle doit effectivement muter et on sait qu'on est cosmopolites,

[32] Entretien, parquet civil, SE. 
mais... [...] je ne sais pas comment on pourra peut-être réagir dans dix, vingt, trente, quarante, cinquante ans, cent ans, je n'en sais rien, si dans le palmarès des prénoms on n'a que des prénoms d'origine musulmane. Je n'ai aucune conviction, je n'ai aucune position, mais est-ce que à un moment donné ça ne va pas faire ressortir des vieilles haines qu'on pourrait éviter, mais dès bien en amont. » (entretien, JAF, SE).

L'autre JAF déplore la sévérité de la juridiction. À propos des demandes motivées par les exigences marocaines, elle se positionne sans équivoque du côté de l'acceptation, fondée sur le respect de la double appartenance nationale. Si on refuse le changement de prénom, " c'est quoi le message? demande-t-elle, leur dire au fond vous n'êtes ni français ni marocain? » La sensibilité à la double appartenance se lit également face aux demandes « identitaires », lorsque la magistrate s'émeut de son aspect douloureux, pour des jeunes tiraillés entre le pays où ils sont nés ou arrivés tout petits et cet autre pays, celui de leurs origines plus lointaines, celui de leurs parents, qu'ils ne connaissent pas, la « communauté » à laquelle ils appartiennent.

Le parquet, puis le tribunal, mettent alors en œuvre des stratégies destinées à concilier, le mieux possible, l'intérêt de l'individu et la préoccupation plus « politique ». Le parquet s'oppose aux demandes motivées par l'État marocain, mais ne fait pas appel des décisions favorables prises par le tribunal. Le tribunal, au lieu de modifier le prénom, joue avec les ajouts de prénoms, le changement de leur ordre.

\section{_" Conclusion}

La justice familiale, lieu privilégié pour l'observation de la « diversité culturelle » ? Sans doute, mais autrement que sous l'angle de la mise en évidence et du traitement de justiciables « différents » par leurs coutumes ou leurs modes de vie. Tel est le premier enseignement, inattendu, de l'enquête : le travail empirique a conduit à une reformulation de son objet. Contrairement au présupposé de départ, la justice familiale n'est pas le lieu où se déploient et sont tranchés des « conflits de normes », entre le droit du pays d'accueil et les systèmes de valeurs de populations venues d'ailleurs. La « diversité » des stéréotypes - que les magistrats autant que les justiciables ont tendance à « passer sous silence » - a fait place, au fil de l'observation des audiences et à travers le regard des magistrats, à la découverte d'une réalité plus complexe. Qu'il s'agisse des couples, lorsqu'ils se défont, ou des individus, lorsqu'ils cherchent à modifier leur identité, la justice familiale met en scène des personnes qui se caractérisent moins par des modes de vie et des valeurs différents de ceux du pays d'accueil que par ce que l'on a qualifié de double appartenance. Les demandes qu'ils forment révèlent moins leur différence que le fait qu'ils se rattachent simultanément à deux univers, aux dimensions multiples - culturelles, géographiques, juridiques. La justice familiale met en scène des gens qui sont d'ici et d'ailleurs, et naviguent, tant bien que mal, entre cet ici et cet ailleurs. La diversité y apparaît comme le produit, complexe et sans cesse en mouvement, du phénomène migratoire.

Quant aux magistrats, eux aussi naviguent, peut-être plus difficilement qu'autrefois, entre deux pôles. Le premier leur est familier : c'est l'examen et le traitement de situations individuelles. En tant qu'individu, le justiciable d'origine immigrée est envisagé sous l'angle de sa double origine et des questions concrètes qu'elle peut poser. Le discours est alors à la fois ouvert et 
pragmatique. Le second pôle, plus problématique, est collectif. Lorsque le propos se généralise, passant de l'immigré à l'immigration, phénomène de masse, il se teinte chez certains de crainte ou de méfiance.

Dans leur pratique, les JAF voient les individus, leur réalité immédiate - et notamment cette dimension particulière de double appartenance - avant ce qu'ils représentent socialement. Accorder ou non une interdiction de sortie du territoire ? La plupart des magistrats privilégient l'importance, pour l'enfant, de maintenir les liens avec ses deux familles. Pour les changements de prénom, accéder aux demandes revient à considérer l'individu et son « intérêt légitime » - bénéficier de ses droits dans son pays d'origine autant que dans le pays d'accueil - et non « ce que cela fait à la société », que ce soit en termes d'image de la société française, de risque de vote populiste ou encore de confrontation entre l'État français et l'État marocain. De la même manière, sur la thématique «mariage et migration » une barrière, invisible mais réelle, existe entre les situations individuelles et ce que le mariage peut représenter en termes de contournement des lois sur l'immigration. «Ce n'est pas de cela que nous sommes juges », disent-elles presque en chœur. Une JAF résume :

"Le jugen'est pas sur une politique publique, il est sur des cas individuels et il faut quand même essayer de s'abstraire, tout en ayant conscience, s'abstraire des contraintes... Je crois que c'est ce qui fait un peu l'honneur du métier, c'est qu'il faut s'abstraire des contraintes, des désagréments, des visions générales. [...] Il me semble que le va-et-vient entre le niveau général et le niveau particulier est extrêmement important, [...] très intéressant. [...] Après, il faut redescendre au niveau particulier et ne pas se laisser polluer par les considérations générales dans un dossier particulier. [...] Mais tous les collègues n'aiment pas ça... C'est vrai qu'on n'est pas beaucoup à accepter de naviguer. „ ${ }^{[33]}$ 